There was a family history of tubercle, but general health fairly good. I diagnosed acute retro-bulbar neuritis, and prescribed usual remedies.

Appended is an account of the progress of the case.

August 10. Paresis of $\mathrm{L}$. sixth nerve.

August 12. Vision of left eye worse. No P.L. Slight blurring of the optic disc. No injection of the eye. Movements limited in every direction, especially outwards. 'Normal temperature. The patient could not smell with the left nostril. On examination by Mr. Lindley Sewell, nothing abnormal in the left nasal cavity. Other cranial nerves normal. No pulsation, no bruit, and nothing felt in the orbit.

Radiogram showed absence of the left frontal sinus.

August 16. Complained of right eye. V. $=5 / 4$. Fundus normal.

August 19. Marked proptosis of the left eye, and some chemosis of the conjunctiva. Left pupil widely dilated and fixed. Movement of the left eye very limited in every direction. R. V. = J. 20. Fundus normal.

August 20. Mr. Lindley Sewell explored the region of the left frontal sinus and exposed the dura, but found no evidence of the sinus or of growth. The left eye was enucleated but no evidence of growth found in the orbit.

August 22. R. eye hand movements on nasal side only. No proptosis, movements good. Slight blurring of optic disc.

August 24 . R. V. $=$ P.L?

The patient died three weeks later, and post mortem a flat sub-dural growth was found, evidently arising in the region of the sella Turcica on the left side, spreading to the middle fossa, and over the small wing of the sphenoid to the right side. The growth showed the characters of a periosteal sarcoma.

\title{
NARROW AND SPIRAL FIELDS OF VISION IN HYSTERIA, MALINGERING, AND NEURASTHENIA
}

BY

\author{
A. F. Hurst, M.A., M.D.Oxon., F.R.C.P., \\ PHYSICIAN AND NEUROLOGIST TO GUY'S HOSPITAL \\ AND \\ J. L. M. Symns, M.A., M.D.Cantab.
}

RETRACTION of the field of vision has been regarded as the most characteristic "stigma" of hysteria since Charcot first drew attention to it in 1872. Janet" considered it to be "the emblem of hysterical sensibility in general," and it led him to describe 
hysteria as a condition due to the "retraction of the field of consciousness." Among ophthalmologists de Schweinitz ${ }^{2}$ regards the sign as a "permanent stigma" of hysteria and regards it as a very valuable aid to diagnosis. But Babinski ${ }^{3}$ who, as chef de clinique, had watched the development of Charcot's views on hysteria, soon felt himself forced to reject them, as he found none of the so-called stigmata in any of a series of 100 consecutive cases of hysteria, examined in great detail by methods which excluded the possibility of suggestion. The distinguished French oculist, Morax ${ }^{4}$ a former assistant of Charcot, from whom he learnt the supposed significance of the retracted field of vision, at first continued to find it in almost every patient suffering from hysterical symptoms. But over twenty years ago he became a convert to

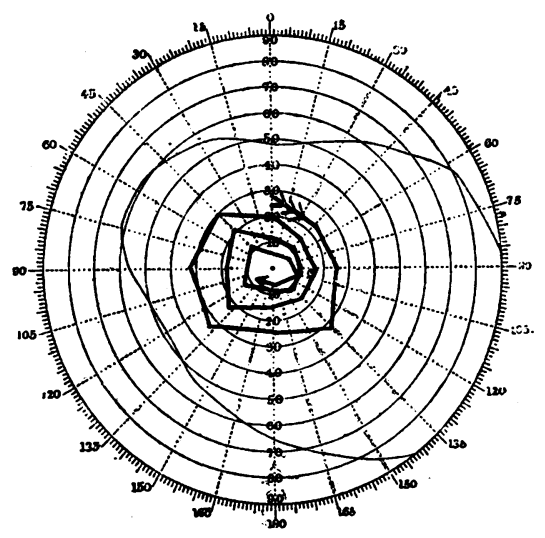

FIG. 1.-Right eye without-inward spiral field of vision in case of hysterical paraplegia.

Babinski's views, and since then he has not seen a single case of hysterical retraction of the field of vision. As it is almost impossible to avoid suggesting a narrow field of vision with the perimeter in highly suggestible patients, he has introduced simpler methods for use with hysterical subjects, in which a finger or other familiar object is employed, and although these appear at first to be less accurate, they. have the great advantage of making it easy to avoid suggesting abnormalities in the field of vision during the examination.

Apart from hysterical amblyopia, we have never seen patients with hysterical symptoms, who spontaneously complained of disabilities resulting from a narrow field of vision, however closely they were cross-examined on the subject. But if a narrow field of vision is produced by testing with the perimeter, the patient may subsequently complain of considerable inconvenience as a result of the cutting off of his peripheral vision. 
We have examined numerous soldiers suffering from various war neuroses, who were abnormally suggestible as a result of the stress and strain of active service, some, but not all, of whom were suffering from gross hysterical symptoms, and we have never found any retraction of their field of vision until they were tested with the perimeter. But the perimeter invariably resulted in the suggestion of a narrowed field, however carefully it was used. Moreover, if the examination was continued after the first field was marked out, a spiral field was always obtained (Fig. 1), identical with that which has hitherto been regarded as a stigma of hysteria (Fig. 2). We believe that the reason why a spiral field of vision, which is the natural result of continued suggestion, has not been found in a larger proportion of hysterical cases showing a narrow field of

(a)
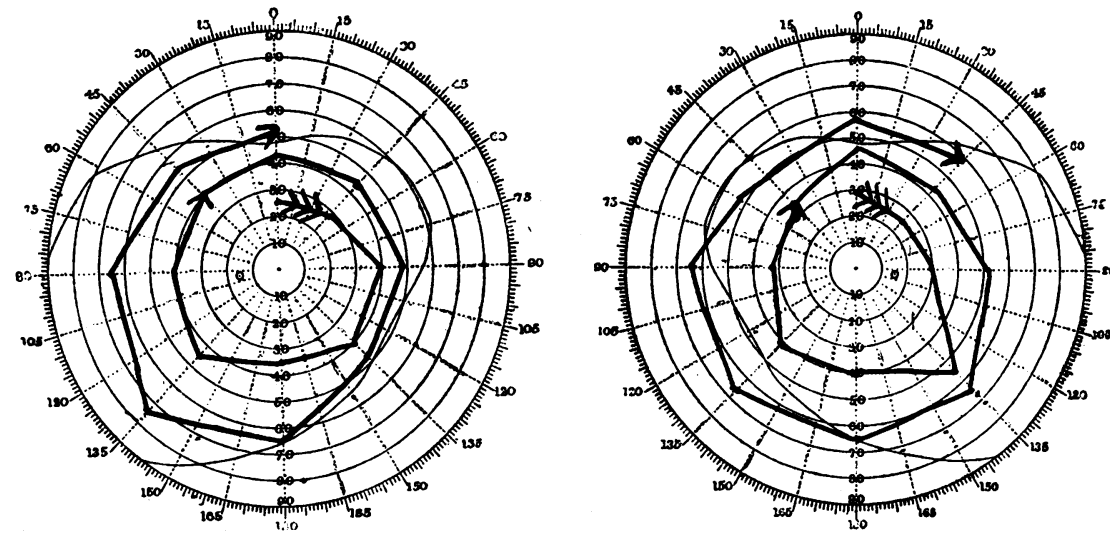

FIG. 3.-Spiral fields in case of hysterical tremor following shell-shock.

(a) LEFT EYE. Within-outward spiral field.

(b) Right Eye. Within-outward spiral field.

vision is simply because it has not been looked for, the examiner being content when he has marked the limit of vision a single time in each direction.

It has generally been taught that a spiral field of vision is a result of fatigue, and it has even been stated that it is more frequently a symptom of neurasthenia than of hysteria. We have found that this is not the case, the inward spiral, which has hitherto alone been described, being a result of the method employed in using the perimeter.* We have found that an outward spiral is always obtained instead of an inward one, if the white disc of the perimeter is moved outwards instead of inwards, as is commonly done (Fig. 3). We have in this way often produced an inward spiral

* In the description of the perimeter given in the four books on ophthalmology, two on neurology, and one on clinical methods which we have consulted, instructions are given to move the disc from without inwards. 
for one eye and an outward one for the other (Fig. 4), or an inward spiral one day and an outward spiral another day with the same eye. There is no question of any special suggestions made by us in our method of using the perimeter, as identical results have been

(a)
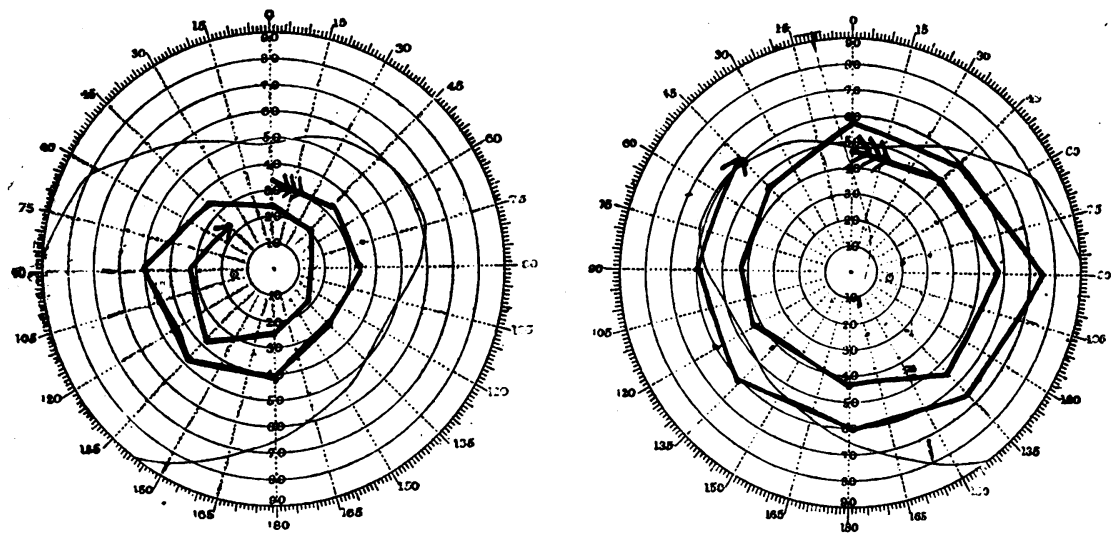

FIG. 4.-Spiral fields in case of hysterical mutism and tremor following shell-shock.

(a) LEFT EYE. Without-inward spiral field.

(b) Right Eye. Within-outward spiral field.

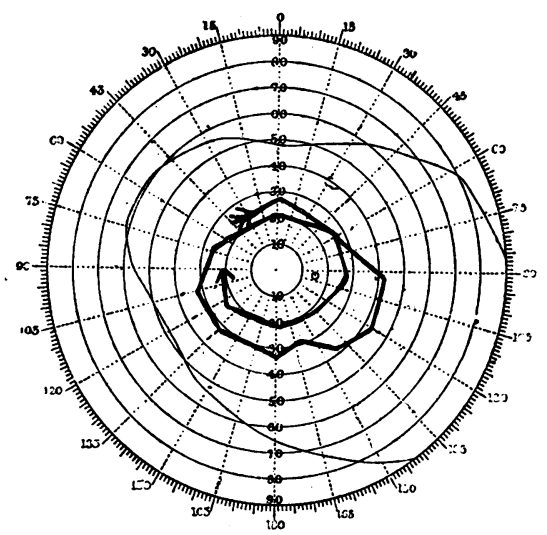

Fig. 5.-Spiral contraction of field of vision in a case of " experimental malingering,",

obtained by other observers, who marked out the fields of vision for us without knowing the nature of the cases or the object we had in view in obtaining the tracings. Moreover, we have always obtained normal fields in normal individuals, whether the disc was moved inwards or outwards.

In the course of some investigations on "experimental" malingering," we asked 27 individuals, who were pretending that they were paralysed on the right side as a result of a railway 
accident, for which they were claiming compensation, whether they could see as well with the right eye as the left. Seven replied that they had noticed some impairment of vision in the right eye. On testing the field of vision with a finger, no narrowing was observed, and they explained that the deficiency they had spoken about was a blurring or general loss of clearness of vision. But when tested with a perimeter all of the seven showed a narrow field on the right side, and one had a slighter narrowing on the left side. In the only two cases in which it occurred to us to continue the investigation after the first field had been marked out a spiral was obtained (Fig. 5), which was identical in character with that supposed to be characteristic of hysteria.

1. P. Janet.-Etat Mental des Hystériques, 1911.

2. de Schweinitz in Posey and Spiller's The Eye and Nervous System, 1906, p. 625.

3. J. Babinski.-Semaine médicale, 1909, Vol. XXIX, p. 3.

4. Quoted by J. Babinski and J. Froment.-Hysterie-Pithiatisme, p. 66, Paris, 1917.

\section{HEREDITARY GLIOMA OF THE RETINA}

BY

H. M. TRAQUAIR, ASSISTANT OPHTHALMIC SURGEON, ROYAL INFIKMARY, EDINBURGH.

IN the Transactions of the Ophthalmological Society of the United Kingdom for 1917, two instances of families affected with hereditary glioma retinae are recorded by Mr. Hill Griffith, who suggests that, in view of their rarity, all such cases should be published.

The following case was recently seen in the clinic under the charge of Dr. J. V. Paterson, at the Edinburgh Royal Infirmary.

The father, A., when six months old, had his left eye removed by the late Argyll Robertson, "for a tumour." Unfortunately, no contemporary record is available, but there can be no doubt that the eye was removed on account of glioma.

A. was married in 1912. Family :

1. Still-birth, "six weeks overdue." 1913.

2. George. 1914. The parents noticed something wrong with the right eye when the child was six weeks old. At eight months, the eye was removed. In June, 1916, glioma of the left eye was apparent, and the orbit was exenterated in 1917. Metastases occurred all over the body, the head became horribly deformed and enlarged, and death supervened in 1918.

3. Mary. 1916. Parents noticed something in the left eye at six weeks. When the child was later brought to the hospital, glioma was diagnosed. Operation was refused and death occurred from broncho-pneumonia soon afterwards. 